\title{
CHARACTERIZATION OF YBCO FILMS BY TORQUE MAGNETOMETRY
}

 \\ Physikalisch-Technisches Institut Jena, Helmholtzweg 4, 0-6900 Jena, Germany (a) and \\ Institut für Angewandte Physik der Universität Regensburg, Universitätsstr. 31, W-8400 \\ Regensburg, Germany (b)
}

\begin{abstract}
YBaCuO films with c- or a-axis texture were prepared by laser deposition and sputtering. From rotational hysteresis losses critical current densities and pinning forces are calculated. The torque relaxation gives activation energies in the range of 0.21 to $0.37 \mathrm{eV}$. Characteristic differences of critical current densities and activation energies are related to the film microstructure.
\end{abstract}

\section{INTRODUCTION}

One of the most challenging problems with high$\mathrm{T}_{C}$ superconducting materials is the understanding of the critical current density in terms of the microstructure. Especially, in the case of quasiepitaxial thin films the pinning effect of different lattice defects is not yet completely understood and, consequently, the relation between deposition parameters and pinning properties of the films is only empirically established. The situation becomes even more complicated by the pronounced anisotropy of the superconducting properties. It was shown recently that torque magnetometry is a useful method for the investigation of superconducting single crystals (e.g. ${ }^{1,2}$ ). In the present paper we report on results obtained by torque measurements of thin films prepared in different ways.

\section{EXPERIMENTAL}

$\mathrm{YBa}_{2} \mathrm{Cu}_{3} \mathrm{O}_{7-x}$ films were deposited on (100) crystals of $\mathrm{SrTiO}_{3}$. We compare here three typical films prepared in the following ways.

Film $A$ was deposited by laser ablation using an excimer laser (308 $\mathrm{nm}, 0.5 \mathrm{~J}, 5 \mathrm{~Hz}$ ). During deposition the substrate was heated up to $750^{\circ} \mathrm{C}$ and the oxygen pressure was kept at 0.25 mbar. Then, the film was cooled down to room temperature in 1 bar oxygen for one hour. The film resistivity is $0.3 \mathrm{~m} \Omega \mathrm{cm}(300 \mathrm{~K})$ and the $T_{c}(R=0)$ is $90 \mathrm{~K}$. It is c- axis textured with a rocking-curve linewidth of the (005) film reflex of $0.7^{\circ}$.

The films $B$ and $C$ were prepared in two steps:

(i) If-planar magnetron sputtering from a ceramic $\mathrm{YBa}_{2.3} \mathrm{Cu}_{3} \mathrm{O}_{7-\mathrm{x}}$ target in argon gas at $10^{-5}$ bar with oxygen of a partial pressure $<10^{-8}$ bar, substrate temperature $<250^{\circ} \mathrm{C}$ and a deposition rate of $1.2 \mu \mathrm{m} / \mathrm{h}$;

(ii) annealing in oxygen ( 1 bar) at a temperature $\mathrm{T}_{\mathrm{a}}$ for ten minutes and cooling down to $\mathrm{T}<300^{\circ} \mathrm{C}$ within 5 hours. Annealing temperature was $900^{\circ} \mathrm{C}$ and $870^{\circ} \mathrm{C}$ for the film $B$ and $C$, respectively.

Film $B$ exhibits prevailing $c$-axis texture with a (005) rocking-curve linewidth of $0.4^{\circ}$ and residual a-axes. Film C shows a-axis texture with a (200) linewidth of $0.6^{\circ}$. The resistivity is $22 \mathrm{~m} \Omega \mathrm{cm}$ and about $10 \mathrm{~m} \Omega \mathrm{cm}$ and the $T_{C}$ values are $85 K$ and $86 \mathrm{~K}$ for the film $B$ and $C$, respectively.

Measurements by means of a sensitive torquemeter were performed for rotation of the magnetic field within a plane either perpendicular or parallel to the film plane. In the latter case the film was carefully oriented with its plane parallel to the field-rotation plane. For fixed field directions the torque relaxation was measured. From in-plane torque curves the pinning force per unit length can be estimated whereas the out-of-plane curves permit the calculation of critical current densities ${ }^{3}$. The experiments were carried out at $79 \mathrm{~K}$ in fields of $16 \mathrm{kA} / \mathrm{m}<\mathrm{H}<640 \mathrm{kA} / \mathrm{m}$. 


\section{RESULTS}

The torque density G/N is shown in Fig. 1 for outof-plane measurements as a function of the angle $\vartheta$ between field and film normal. Figure 2 gives $\mathrm{G} N$ curves for in-plane measurements as a function of the azimuth $\varphi$. Both figures are taken for fields in the range $\mathrm{H}_{\mathrm{c} 1} \ll \mathrm{H} \ll \mathrm{H}_{\mathrm{C} 2}$.

For field rotation in a plane perpendicular to the film plane (Fig.1) we observe torque extrema near $\vartheta=90^{\circ}$ which are considerably different with respect to both shape and magnitude for the three films.

Under the assumption that the magnetization is perpendicular to the film plane ${ }^{1}$ Hagen et al. ${ }^{3}$ calculated the critical current density $\mathrm{j}_{\mathrm{C}}$ from the torque difference $\Delta G$ in increasing and decreasing field $\mathrm{H}$ at a fixed angle $\vartheta$ according to

$$
\mathrm{j}_{\mathrm{C}}=3 \Delta \mathrm{G} /\left(2 \mathrm{RV} \mu_{\mathrm{o}} \mathrm{H} \sin \vartheta\right)
$$

where $R$ and $V$ mean the radius and the volume of the film, respectively. As proved $i^{4}$ the same $j_{C}$ value is obtained if $\Delta G$ is taken from the torque curves for clockwise and counter-clockwise rotation in a constant external field. The latter method was used to derive the results of Fig. 3 in dependence of the field direction. Fig. 4 shows the torque relaxation after stopping the field rotation at an angle $\vartheta=\vartheta_{0}$ in a logarithmic time scaling.

\section{DISCUSSION}

Detailed investigations of $\mathrm{YBa}_{2} \mathrm{Cu}_{3} \mathrm{O}_{7-x}$ crystals revealed spike shaped torque extrema in each case when the field direction is crossing the CuO planes ${ }^{2}$ in accordance with a model published by Tachiki and Takahashi ${ }^{5}$. The torque spikes at $\vartheta=90^{\circ}$ in Fig. $1 \mathrm{a}$ and $1 \mathrm{~b}$ can be ascribed to the same effect since the films $A$ and $B$ are c-textured with their $\mathrm{CuO}$ planes parallel to the film plane. This conclusion is supported by the absence of any spike in the torque curve of film $C$ (Fig. 1C). The spikes are superimposed on sine shaped background losses which may be explained by assuming a nearly rectangular hysteresis loop $\mathrm{M}(\mathrm{H})$ perpendicular to
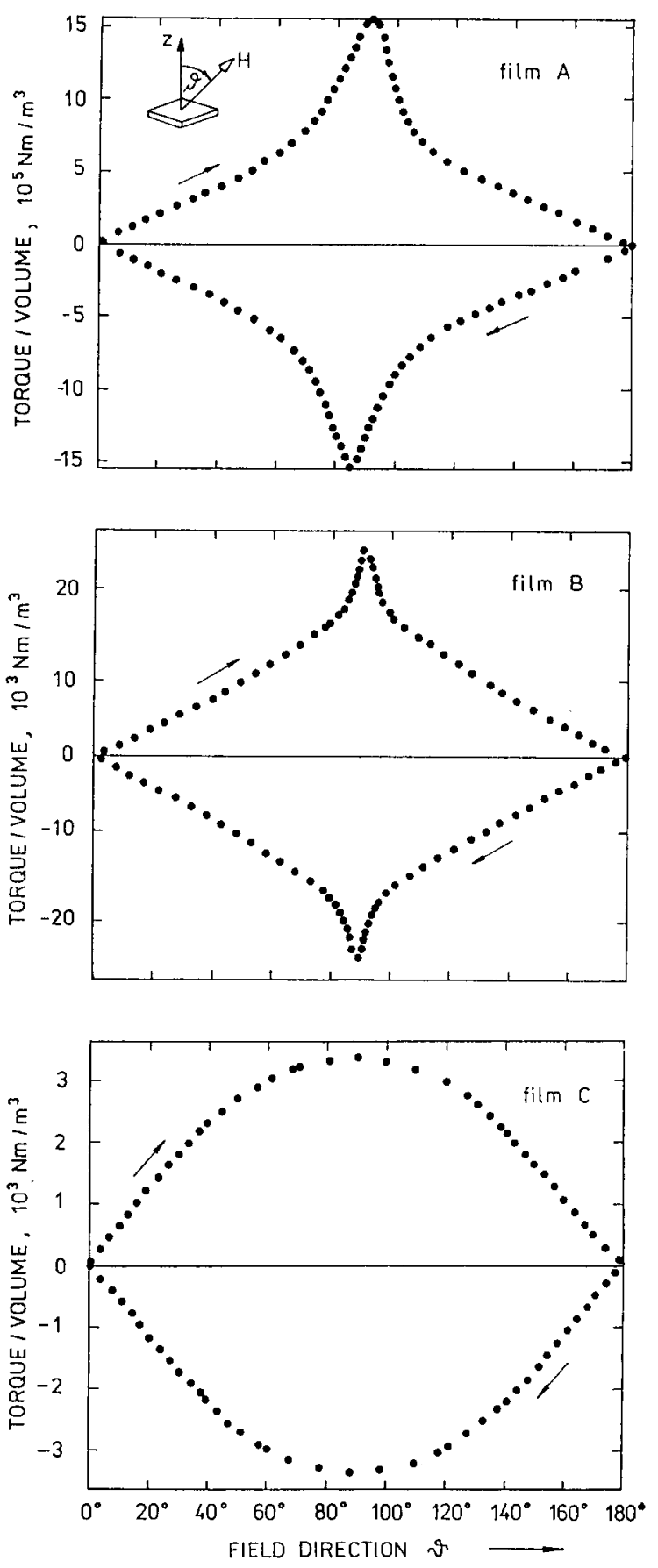

FIGURE 1

Torque curves for rotation of the external magnetic field $\left(\mathrm{H}=4 \times 10^{5} \mathrm{~A} / \mathrm{m}\right)$ perpendicular to the film plane for films $A, B$ and $C(T=79 K)$ 



FIGURE 2

Torque curves for rotation of the external magnetic field $\left(\mathrm{H}=4 \times 10^{5} \mathrm{~A} / \mathrm{m}\right)$ within the film plane for films $\mathrm{A}$, $B$ and $C(T=79 \mathrm{~K})$ the film plane and a neglegible magnetization component parallel to the film plane ${ }^{1}$.

Fuhrmans and Heiden ${ }^{6}$ gave a relationship between the pinning force per unit length of a vortex $f_{p}$ and the irreversible in-plane torque $G_{i r r}=\left(G^{+}-G^{-}\right) / 2$ :

$$
f_{p}=3 \pi G_{i r r} \phi_{o} /\left(4 \mu_{0} H V R\right)
$$

where $\mathrm{G}^{+}$and $\mathrm{G}^{-}$mean the torque measured during clockwise and counter-clockwise field rotation, respectively. $\phi_{0}$ is the flux quantum, $V$ and $R$ are the volume and the radius of the sample. The films exhibit nearly isotropic behaviour with respect to the angular dependence of $G_{i r r}$. However, the reversible torque contribution $\mathrm{G}_{\mathrm{rev}}=\left(\mathrm{G}^{+}+\mathrm{G}^{-}\right) / 2$ is clearly anisotropic for the film $C$ with a fourfold periodicity exhibiting two easy axes which are located at the two possible orientations of the $\mathrm{CuO}$ planes.

Applying formula (2) to film A of Fig. 2 we get $f_{p}=1.5 \times 10^{-9} \mathrm{~N} / \mathrm{m} .7$ However, in contrast to Fuhrmans and Heiden who considered rather thick cylindrical plates, we have to take into account the

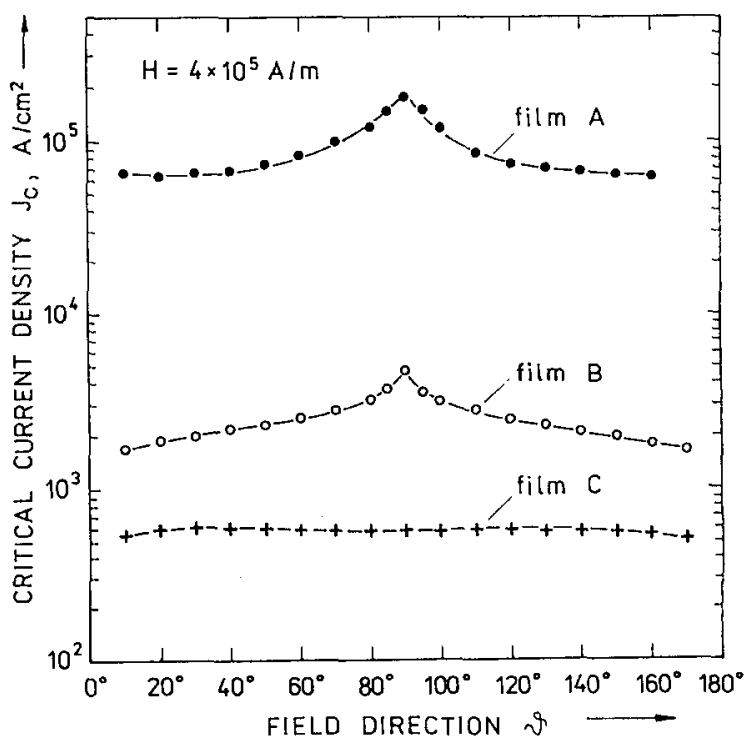

FIGURE 3

Dependence of critical current density on field direction ( $T=79 \mathrm{~K}$ ) 




FIGURE 4

Relaxation of the torque at $79 \mathrm{~K}$ for films $\mathrm{A}, \mathrm{B}$ and $\mathrm{C}$

influence of the London-penetration depth for which the value at $79 \mathrm{~K}$ was used for all films regardless of their different $T_{c}$ values. The corrected ${ }_{f}$ values are given in the following table:

\begin{tabular}{llll} 
Film & $\begin{array}{c}\lambda_{0}{ }^{8} \\
(\mu \mathrm{m})\end{array}$ & $\begin{array}{l}\mathrm{D} \\
(\mu \mathrm{m})\end{array}$ & $\begin{array}{l}\text { corrected } \mathrm{f}_{\mathrm{p}} \\
(\mathrm{N} / \mathrm{m})\end{array}$ \\
\hline $\mathrm{A}$ & 0.45 & 0.6 & $2.7 \cdot 10^{-8}$ \\
$\mathrm{~B}$ & 0.45 & 1.9 & $8.0 \cdot 10^{-10}$ \\
$\mathrm{C}$ & 0.13 & 1.5 & $1.7 \cdot 10^{-10}$
\end{tabular}

However, these $f_{p}$ values are yet much smaller than values calculated from critical current densities. The critical current densities (Fig. 3) determined by means of equation (1) are an order of magnitude smaller than the values obtained with transport measurements. This discrepancy was observed already by Fischer et al. ${ }^{9}$ for $\mathrm{j}_{\mathrm{C}}$ values derived from magnetization curves. The angular dependence of $\mathrm{j}_{\mathrm{C}}$ shows typical maxima at $\vartheta=90^{\circ}$ which are also found with transport measurements by Roas et al. ${ }^{10}$. The curve of film $C$ shows no maximum in accordance with the explanation of the $j_{C}$ spikes by CuO-plane pinning.

Griessen et al. ${ }^{11}$ calculated the activation energy $U$ for flux motion from the magnetization relaxation. The corresponding formula for the torque is

$$
\frac{G\left(t-t^{\prime}\right)}{G\left(t^{\prime}\right)}=\frac{1-(k T / U) \ln \left[\left(t-t^{\prime}\right) / \tau\right]}{1-(k T / U) \ln \left(t^{\prime} / \tau\right)}
$$

Here, $t^{\prime}$ means the time for the beginning of a nearly logarithmic relaxation. Taking for $\tau$ a possible range of $10^{-12} \mathrm{~s}<\tau<10^{-6} \mathrm{~s}^{11}$ we get the activation energies $(0.37 \pm 0.05) \mathrm{eV},(0.23 \pm 0.05) \mathrm{eV}$ and $(0.21 \pm 0.05) \mathrm{eV}$ for film $\mathrm{A}, \mathrm{B}$ and $\mathrm{C}$, respectively. For $t<t$ there are considerable differences between the relaxation curve of film $\mathrm{A}$ and the two other curves. The latter exhibit during the first seconds a rather strong decrease which may be explained by the existence of many shallow pinning valleys in the films.

1. C. Giovanella, L. Fruchter, G. Collin, and I.A. Campbell, Physica C 153-155 (1988) 1473.

2. R. Hergt, W. Andrä, and W. Schüppel, phys. stat. sol. (a) 123 (1991) 255.

3. C.W. Hagen, M.R. Bom, R. Griessen, B. Dam, and $H$. Veringa, Physica C 153-155 (1988) 322.

4. W. Andrä, T. Eick, R. Hergt, W. Michalke, W. Schüppel, and K. Steenbeck, this conference.

5. M. Tachiki and S. Takahashi, Solid State Com. 70 (1989) 291.

6. M. Fuhrmans and C. Heiden, Cryogenics 16 (1976) 451.

7. W. Andrä, J. Betz, B. Brunner, R. Hergt, H. Lengfellner, K.F. Renk, W. Schüppel, and K. Steenbeck, phys. stat. sol. (a) 122 (1990) K161.

8. L. Krusin-Elbaum, A.P. Malozemoff, Y. Yeshurun, D.C. Cronemeyer, and F. Holtzberg, Phys. Rev. 39 (1989) 2936.

9. P. Fischer, H.W. Neumüller, B. Roas, H.F. Braun, and G. Saemann-Ischenko, Solid State Com. 72 (1989) 871.

10. B. Roas, L. Schultz, and G. Saemann-Ischenko, Phys. Rev. Letters 64 (1990) 479.

11. R. Griessen, C.F.J. Flipse, C. Hagen , J. Lensink, B. Dam, and G.M. Stollman, J. Less-Common Metals 151 (1989) 39. 